\title{
A Self-Management App for Patients with Schizophrenia: A Pilot Study
}

\author{
Buket CINEMRE ${ }^{\mathrm{a}}$, Mahmut GULERCE ${ }^{\mathrm{b}, 1}$ and Kemal Hakan GULKESEN ${ }^{\mathrm{b}}$ \\ a Department of Psychiatry, Faculty of Medicine, Akdeniz University, Antalya, Turkey \\ ${ }^{\mathrm{b}}$ Department of Biostatistics and Medical Informatics, Faculty of Medicine, Akdeniz \\ University, Antalya, Turkey
}

\begin{abstract}
We aimed to observe the effects of a self-management mobile app for patients with schizophrenia. A mobile app was designed to record and remind users to take medication and some daily activities. The patients were asked to use the app for one month. Before starting to use the app, and after completion of one month, the patients were assessed by some psychiatric scales. Twelve patients completed the study. The mean number of automatic reminders per patient was $918 /$ month or $29.6 /$ day. The mean entry number per patient was 158.4 month or $5.1 /$ day. The numbers of daily recorded activities showed a decreasing tendency over time. The pre-study PANSS score was $16.2 \pm 5.8(\operatorname{mean} \pm \mathrm{SD})$ and the post-study score was $14.9 \pm 6.9(\mathrm{p}=0.040)$. The pre-study FROGS daily life skills score was $20.3 \pm 3.8$ and the post-study score was $19.7 \pm 4.1(\mathrm{p}=0.012)$. The results suggest that a mobile app may be helpful for patients with schizophrenia.
\end{abstract}

Keywords. Schizophrenia, smartphone, mobile applications, reminder systems

\section{Introduction}

Treatment of schizophrenia is mainly pharmacological, however, patient non-adherence to medication is a serious problem. Three-fourths of patients with schizophrenia become non-adherent within two years of hospital discharge [1]. In addition to pharmacological treatment, psychosocial treatments have been suggested for the treatment of schizophrenia. Some of the evidence-based practices include illness self-management training, and social skills training [2]. Mobile phones may support adherence to taking medication and psychological interventions. In a review on apps to support coordinated specialty care for prodromal and early course schizophrenia disorders, the authors found 21 eligible studies on 16 apps. Only three of these studies were intervention studies [3].

The primary objective of the present study is to observe if a self-management mobile app for patients with schizophrenia affects their Positive and Negative Syndrome Scale (PANSS) [4] score after one month of use. The secondary objective is to observe their mobile app usage patterns.

\footnotetext{
${ }^{1}$ Corresponding Author, Mahmut GULERCE, Department of Biostatistics and Medical Informatics,
} Faculty of Medicine, Akdeniz University, 07070 Antalya, Turkey; E-mail: mahmutta@gmail.com. 


\section{Methods}

The patient activities were divided into four groups; 1) Self-care activities (eating, shaving, taking shower, make-up, etc.), 2) Household duties (cooking, cleaning, shopping, etc.), 3) Social activities (meeting with friends, calling friends, forming a new acquaintance, etc.), and 4) Drug adherence. In the following meetings, detailed lists of activities under each group were prepared. The system was designed with three components 1) Database and core software, 2) Mobile app, and 3) Web interface for physicians. The mobile app has the function of sending reminders for each activity and recording performed activities based on input from the patients. The physicians could adjust the activities for each individual patient.

The operating system of the web server was CentOS v6.5. Apache webserver 2.2, MySql database server service 5.7.21, and some 1st part libraries for PHP programming language were installed on the core component. The system was hosted on a cloud-based VPS server. The app was developed on the Apache Cordova development platform, which provides a single development environment for Android, IOS and Windows operated mobile phones. We did not use a third-party service for notification messages (reminders) because of concerns about security. We developed an isolated notification engine and integrated it into the system. The patient data was not stored in the patients' smartphones. All the communications between the app, the physician interface and the server were secured by an SSL certificate. The physician user interface was restricted by IP control.

Before the study, usability tests for the app were performed. Heuristic evaluation was completed by five experts. After re-designing the app according to the results of the heuristic evaluation, we performed a second usability test with five patients with schizophrenia using the think-aloud method. The app was re-designed after the second step of the usability study.

There was a default activity list for the patients. They were expected to have breakfast every day, go shopping every three days, clean the home every week, etc. The physician could tailor the default activities and add new activities for each patient. For example, if the patient had a daily shaving habit, it was also added to the patient's activity list. The medications were also entered by the physician. The patients could see their activity list in the mobile app and they were expected to record their activities (Figure 1). If they did not enter an activity in the expected period, the mobile app reminded them.

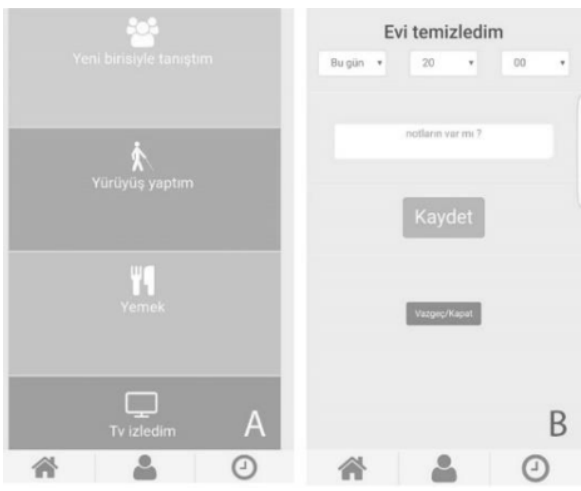

Figure 1. Activity menu (A), and activity record screen (B) of the app. 
The study included patients with a diagnosis of schizophrenia according to the DSMV, who satisfied the following criteria: age 18-65 years; active smartphone users; clinically stable patients who regularly visited our outpatient center over the previous three months. All patient treatments, including medication types and doses, were maintained for the duration of the study period. The ethical permission was obtained from the Akdeniz University Clinical Research Ethical Committee (2015/130). The number and type of reminders were tailored for each patient with the help of the web interface. The patients were asked to use the app for one month after user training. They were given a telephone number to contact in case of technical problems, but no additional support or stimulation was provided except smartphone reminders.

Before they began using the app, and after completion of one month, the psychopathological status of the patients was assessed by the PANSS (used for measuring symptom severity of patients with schizophrenia), the Clinical Global Impressions Scale (CGI, used for measuring symptom severity, treatment response and the efficacy of treatments in treatment studies of patients with mental disorders) [5], the Instrumental Activities of Daily Living (IADL) Scale (evaluates the activities that allow an individual to live independently in a community) [6], and The Functional Remission of General Schizophrenia (FROGS) Scale (it is specific to schizophrenia, which assesses daily and social functioning comprehensively, regardless of the psychotic symptoms) [7]. We recruited 14 patients into the study, but we lost two of them in the follow-up. So, we completed the study with 12 patients, resulting in a 0.71 post-study power for detection of the difference in 16 (Cohen's d: 0.8) of the PANNS score. Data were compared using paired t-test or the Wilcoxon test. Spearman's rho test was used for correlation analysis.

\section{Results}

One patient used the app less than 30 days and one patient did not participate in poststudy tests, so we completed the study with 12 patients. On average, the patients were $34.6 \pm 10.8$. Eight $(66.7 \%)$ of them were female and four $(33.3 \%)$ of them were male. In one month, 12 patients recorded a total of 1901 activities. The mean of automatic reminders per patient was $918 /$ month or 29.6 /day. The mean entry number per patient was 158.4 (median 116.5) and for each patient, the mean entry number per day was 5.1. The number of recorded activities of the patients in the study showed great variation (minimum 22, maximum 388). The most frequent entries were; Ate $171(9.0 \%)$, washed my face $163(8.6 \%)$, brushed my teeth $140(7.4 \%)$, had breakfast $114(6.0 \%)$, and made my bed $104(5.5 \%)$. We observed an ongoing resistance to recording their medication. The average monthly entry for each patient is only five times.

The numbers of daily recorded activities showed a decreasing tendency over time (Figure 2). Pre-study and post-study test results of the patients are shown in Table 1.

\section{Discussion and Conclusion}

The most striking result of our study is the observation of the patients' decrease in app use over time. Despite being a relatively young group of participants, the patients used the app an average of 5.1 times a day. This number is similar to 5.2 in FOCUS [8] and 4.2 in A4i studies [9]. Better adherence in the first week of the study in patients with schizophrenia was also observed in other previous studies $[10,11]$. The app produced a 
mean of 918 reminders for each patient and they responded to $17.3 \%$ of them. This behavior reminds us of "alert fatigue", a phenomenon that is discussed for clinical decision support systems [12].

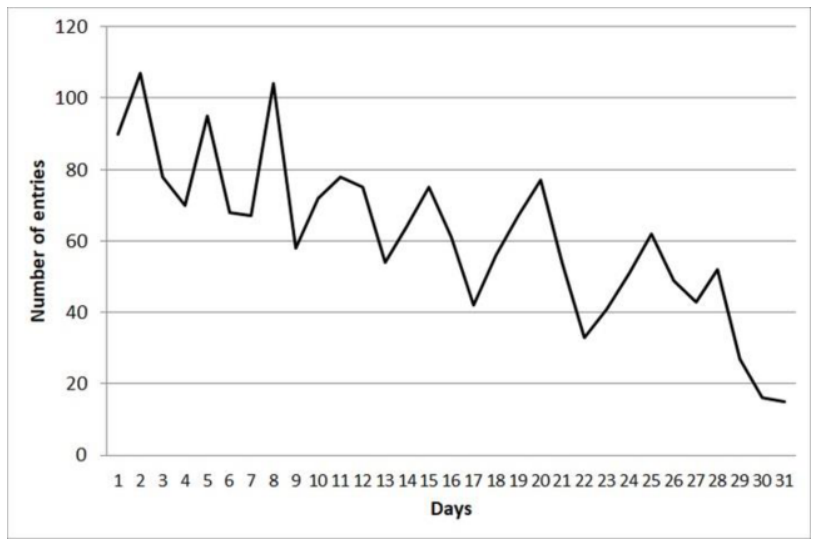

Figure 2. The number of daily activity records during usage of the patients for 31 days.

Table 1. Pre-study and post-study test results of the patients *Data with parametric distribution is shown as mean \pm standard deviation, and data with non-parametric distribution are shown as median (Q1-Q3).

\begin{tabular}{lrrr}
\hline \multicolumn{1}{c}{ Test } & Pre-study* & Post-study $^{*}$ & p \\
\hline PANSS positive signs & $16.2 \pm 5.8$ & $14.9 \pm 6.9$ & 0.040 \\
PANSS negative signs & $16.0 \pm 5.5$ & $15.1 \pm 5.6$ & 0.374 \\
PANSS general psychopathology scale & $29.3 \pm 7.2$ & $27.8 \pm 11.6$ & 0.409 \\
PANSS Total & $61.4 \pm 16.4$ & $57.8 \pm 20.0$ & 0.103 \\
FROGS social functioning & $17.8 \pm 4.5$ & $17.7 \pm 4.5$ & 0.809 \\
FROGS health and treatment & $13.3 \pm 2.8$ & $13.3 \pm 2.9$ & 0.586 \\
FROGS daily life skills & $20.3 \pm 3.8$ & $19.7 \pm 4.1$ & 0.012 \\
FROGS occupational functioning & $5.8 \pm 1.8$ & $5.6 \pm 2.0$ & 0.339 \\
FROGS Total & $56.9 \pm 11.6$ & $56.2 \pm 12.3$ & 0.069 \\
CGI Severity of illness & $3(2-4)$ & $3(2-4)$ & 0.157 \\
IADL total & $7.5(7.0-8.0)$ & $7.5(7.0-8.0)$ & 0.317 \\
\hline
\end{tabular}

Previous studies showed a positive effect of the mobile intervention on patients with schizophrenia [8-9, 13-14]. In the present study, when pre-study and post-study scores of PANNS, FROGS, CGI, and IADL were compared, statistical significance was observed only for PANNS positive signs (mean pre-test 16.2 and post-test 14.9) and FROGS daily life skills (mean pre-test 20.3 and post-test 19.7). Interestingly, the PANNS positive signs score shows improvement while the FROGS daily life skills score shows deterioration during the study period. The general effect seems to be positive because the size effect of PANNS positive signs is larger than FROGS daily life skills score, but due to the small sample size and absence of a control group, these findings must be carefully interpreted. These changes may be random or a result of other factors such as medication.

A major limitation of our study is our small number of patients. The study was originally planned for 14 patients, but we finished the study with 12 patients. This number would be moderate in case of good patient adherence, but we observed an apparent decrease in patient usage during the one-month study period. Because of the decreasing usage problem, we are suspicious if pre and post-test comparisons reflect the 
real effect of such an app. Another limitation is the absence of a control group. The observed difference in test scores may be due to the natural course of the disease or result of the patients' medication. The absence of a post-study questionnaire also limits the value of the present study. A feedback questionnaire would add some detailed information on the user perspective and experience.

The results of the present study show a decrease in time of usage of an app for selfmanagement by patients with schizophrenia. A reason for this problem may be the high number of reminders produced by the app. With the help of a more carefully selected smaller set of reminders, better adherence to the app may be obtained. Another approach may be the addition of some data which are automatically produced by smartphones. Modern smartphones carry several sensors including localization and acceleration sensors which can produce valuable data about the patient activity. We also observed quite variable usage patterns among patients. In future studies, larger groups would be helpful to stratify patients to observe different usage patterns and understand the type of patients who will benefit from the intervention. A control group would also be very helpful to isolate the effect of intervention from other possible factors.

\section{References}

[1] Cañas F, Alptekin K, Azorin JM, et al. Improving treatment adherence in your patients with schizophrenia: the STAY initiative. Clin Drug Investig. 2013 Feb;33(2):97-107.

[2] Mueser KT, Deavers F, Penn DL, Cassisi JE. Psychosocial treatments for schizophrenia. Annu Rev Clin Psychol. 2013;9:465-97.

[3] Camacho E, Levin L, Torous J. Smartphone Apps to Support Coordinated Specialty Care for Prodromal and Early Course Schizophrenia Disorders: Systematic Review. J Med Internet Res. 2019 Nov 12;21(11):e16393.

[4] Kay SR, Opler LA, Lindenmayer JP. The Positive and Negative Syndrome Scale (PANSS): rationale and standardisation. Br J Psychiatry Suppl. 1989 Nov;(7):59-67.

[5] Busner J, Targum SD. The clinical global impressions scale: applying a research tool in clinical practice. Psychiatry (Edgmont). 2007 Jul;4(7):28-37.

[6] Lawton MP, Brody EM. Assessment of older people: self-maintaining and instrumental activities of daily living. Gerontologist. 1969 Autumn;9(3):179-86.

[7] Llorca PM, Lançon C, Lancrenon S, Bayle FJ, Caci H, Rouillon F, Gorwood P. The "Functional Remission of General Schizophrenia" (FROGS) scale: development and validation of a new questionnaire. Schizophr Res. 2009 Sep;113(2-3):218-25.

[8] Ben-Zeev D, Kaiser SM, Brenner CJ, et al. Development and usability testing of FOCUS: a smartphone system for self-management of schizophrenia. Psychiatr Rehabil J. 2013 Dec;36(4):289-296

[9] Kidd SA, Feldcamp L, Adler A, et al. Feasibility and outcomes of a multi-function mobile health approach for the schizophrenia spectrum: App4Independence (A4i). PLoS One. 2019;14(7):e0219491.

[10] Palmier-Claus J, Rogers A, Ainsworth J, et al. Integrating mobile-phone based assessment for psychosis into people's everyday lives and clinical care: a qualitative study. BMC Psychiatry. 2013 Jan 23;13:34.

[11] Ainsworth J, Palmier-Claus J, Machin M, et al. A comparison of two delivery modalities of a mobile phone-based assessment for serious mental illness: native smartphone application vs text-messaging only implementations. J Med Internet Res. 2013 Apr 05;15(4):e60.

[12] Ancker JS, Edwards A, Nosal S, et al. Effects of workload, work complexity, and repeated alerts on alert fatigue in a clinical decision support system. BMC Med Inform Decis Mak. 2017 Apr 10;17(1):36.

[13] Ben-Zeev D, Brenner CJ, Begale M, et al. Feasibility, acceptability, and preliminary efficacy of a smartphone intervention for schizophrenia. Schizophr Bull. 2014 Nov;40(6):1244-1253.

[14] Schlosser DA, Campellone TR, Truong B, Etter K, Vergani S, Komaiko K, Vinogradov S. Efficacy of PRIME, a Mobile App Intervention Designed to Improve Motivation in Young People With Schizophrenia. Schizophr Bull. 2018 Aug 20;44(5):1010-1020. 